ORIGINAL ARTICLE

\title{
Neonatal cranial ultrasound versus MRI and neurodevelopmental outcome at school age in children born preterm
}

\author{
K J Rademaker, C S P M Uiterwaal, F J A Beek, I C van Haastert, A F Lieftink, \\ F Groenendaal, D E Grobbee, L S de Vries
}

See end of article for authors' affiliations

.....................

Correspondence to: Linda S de Vries,

Department of

Neonatology,

KE 04.123.1, University Medical Centre Utrecht/ Wilhelmina Children's Hospital, PO Box 85090, 3508 AB Utrecht, The Netherlands; I.devries@ wkz.azu.nl

Accepted 20 May 2005

Published Online First 14 June 2005

\begin{abstract}
Aim: To examine the correlation between neonatal cranial ultrasound and school age magnetic resonance imaging (MRI) and neurodevelopmental outcome.

Methods: In a prospective 2 year cohort study, 221 children (gestational age $\leqslant 32$ weeks and/or birth weight $\leqslant 1500 \mathrm{~g}$ ) participated at a median age of 8.1 years (inclusion percentage $78 \%$ ). Conventional MRI, IQ (subtests of the WISC), and motor performance (Movement Assessment Battery for Children) at school age were primary outcome measurements.

Results: Overall, there was poor correspondence between ultrasound group classifications and MRI group classifications, except for the severe group (over $70 \%$ agreement). There was only a $1 \%$ chance of the children with a normal cranial ultrasound having a major lesion on MRI. Mean IQ (standard deviation) was significantly lower in children with major ultrasound or MRI lesions, but was also lower in children with minor lesions on MRI compared to children with a normal MRI $191 \pm 16,100 \pm 13,104 \pm 13$ for major lesions, minor lesions, and normal MRI, respectively). Median total impairment score (TIS) was significantly higher in children with major lesions on ultrasound or MRI as well as in children with minor lesions on MRI (TIS 4.0 and 6.25 for normal and minor lesions on MRI, respectively; $p<0.0001$ ).

Conclusions: A normal neonatal cranial ultrasound excluded a severe lesion on MRI in $99 \%$ of cases. MRI correlated more strongly with mean IQ and median TIS than ultrasound. Subtle white matter lesions are better detected with MRI which could explain the stronger correlation of MRI with IQ and motor performance.
\end{abstract}

$\mathrm{N}$ eonatal cranial ultrasound (US) remains the method of choice to detect brain injury in high risk preterm infants on neonatal intensive care units (NICU). It is a non-invasive, inexpensive bedside tool and examinations can be repeated as often as necessary without major disturbance of vulnerable infants. The anterior and posterior fontanelles form excellent acoustic windows to examine the deep midline and periventricular regions of the brain. ${ }^{1}$ We recently demonstrated that cerebral palsy (CP) can be accurately predicted with US. ${ }^{2}$ However, cranial US is not very suitable for investigating cortical regions or structures in the posterior fossa. ${ }^{3}$

Over the last decades, magnetic resonance imaging (MRI) has increasingly become available. It provides high resolution, non-invasive imaging of the brain and all areas can be easily visualised. ${ }^{4}$ However, it requires transportation of sometimes critically ill children to an MRI unit, is time consuming, and is not available on a routine basis.

Good correlation between US and neonatal MRI was found for haemorrhages and ventriculomegaly. ${ }^{5}$ Agreement between US and MRI was also good for cystic periventricular leukomalacia (PVL), but for more subtle white matter injury MRI proved to be far more sensitive. ${ }^{6-10}$

The aim of the present study was to compare neonatal cranial US with school age MRI and to investigate whether a normal US excludes major lesions on future MRI. We also investigated which imaging technique correlated best with two parameters of neurodevelopmental outcome.

\section{METHODS}

The children described in this paper are part of a cohort of patients consecutively admitted soon after birth to the NICU of Wilhelmina Children's Hospital, a tertiary referral hospital. All 375 children born between March 1, 1991 and March 1, 1993 with a gestational age (GA) of $\leqslant 32$ weeks (range 2533 ) and/or a birth weight (BW) of $\leqslant 1500 \mathrm{~g}$ were enrolled in a long term follow up study. Sixty four children (17\%) died and $28(7.5 \%)$ were excluded because of (multiple) congenital abnormalities and/or chromosomal disorders. At the age of 7 or 8 , the children were invited to the hospital to undergo several tests (WISC, ${ }^{11}$ Movement Assessment Battery for Children (ABC), ${ }^{12}$ and brain MRI). Of the remaining 283 children, $22(7.8 \%)$ could not be traced and the parents of $25(8.8 \%)$ refused participation. As a result, 236 children $(83.4 \%)$ took part in the study.

Neonatal cranial US results were available in 234 of the 236 children. MRI could not be completed because of anxiety in 10 children. Children with a congenital abnormality $(n=2)$ on US were excluded as well as one child who developed an unknown neuromuscular disorder. This resulted in a total study population of 221 children (78\% of the original cohort). Mean GA of the children was 29.4 weeks (standard deviation (SD) 2.0) and mean BW $1197 \mathrm{~g}$ (SD 315). In this group 54.8\% were male and $45.2 \%$ female.

\footnotetext{
Abbreviations: $\mathrm{BW}$, birth weight; $\mathrm{CP}$, cerebral palsy; DEHSI, diffuse excessive high signal intensity; FLAIR, fluid attenuated inversion recovery; GA, gestational age; IR, inversion recovery; $\mathrm{IVH}$, intraventricular haemorrhage; MRI, magnetic resonance imaging; NICU, neonatal intensive care unit; PHVD, post haemorrhagic ventricular dilatation; PVL, periventricular leukomalacia; SD, standard deviation; TIS, total impairment score; TSE, turbo spin echo; US, ultrasound; VD, ventricular dilatation
} 
The Medical Ethics Committee of the University Medical Centre Utrecht approved the study and parental informed consent was obtained.

\section{Cranial US}

Cranial US was performed within $6 \mathrm{~h}$ after admission, at least three times during the first week, subsequently once a week until discharge, and again at term age. A standardised protocol of acquiring images (coronal, midsagittal, and parasagittal planes) was used. Infants were examined with an ATL UM-4 mechanical sector scanner (Philips Medical Systems, Best, The Netherlands) using the 7.5 MHz transducer to ensure the best possible resolution.

All US scans were analysed by one neonatologist (LDV), who at that time could not be aware of later MRI findings and outcome. US scans were classified according to the most severe lesions seen at any time.

Intraventricular haemorrhages (IVH) were graded according to Papile et al ${ }^{13}$ and PVL according to de Vries et al. ${ }^{14}$ Neonatal US scans were also analysed for the presence of ventricular dilatation (VD; post hemorrhagic or ex vacuo), calcifications, germinal layer necrosis, germinal layer/choroid plexus cysts, or subependymal pseudocysts.

Cranial US findings were classified into three groups: normal when no or minor abnormalities, such as germinal layer/plexus cysts, subependymal pseudocysts, or calcifications as exclusive findings were present (group 1); mildly abnormal when an IVH grade I/II, PVL I, or germinal layer necrosis, or a combination of these features, was present (group 2); and severely abnormal when one or more of the following features were present: IVH grade III/IV, cystic PVL II/III, thalamic lesion, focal infarction, or convexity haemorrhage (group 3).

VD as a single feature was assigned to the mildly abnormal group. VD following a haemorrhage (PHVD) with need for therapeutic intervention was listed in the severely abnormal group.

\section{MRI}

MRI was performed without sedation using a 1.5 T Philips Gyroscan ACS-NT system (Philips Medical Systems, Best, The Netherlands). Images were acquired with a $256 \times 256$ matrix and a field of view of $230 \mathrm{~mm}$ and included a sagittal $\mathrm{T}_{1}$ survey (TR/TE 512/15 ms; slice thickness $4.0 \mathrm{~mm}$, interslice gap $0.6 \mathrm{~mm}$ ), transverse dual turbo spin echo (TSE) images (first echo TR/TE 4000/17 ms; second echo TR/TE 4000/ $110 \mathrm{~ms}$; slice thickness $5.0 \mathrm{~mm}$, interslice gap $1.0 \mathrm{~mm}$ ), transverse $T_{1}$ inversion recovery (IR) TSE images (TR/TE/TI 2346/10/300 ms; slice thickness $5.0 \mathrm{~mm}$, interslice gap $1.0 \mathrm{~mm}$ ), and coronal $\mathrm{T}_{2}$ fluid attenuated inversion recovery (FLAIR) images (TR/TE/TI 7262/100/2000 ms; slice thickness $5.0 \mathrm{~mm}$, interslice gap $1.0 \mathrm{~mm}$ ).

All MRI scans were assessed by one radiologist (FJAB), blinded to neonatal US findings and outcome. MRI images were scored for the presence of periventricular gliosis on coronal FLAIR images, VD, corpus callosum thinning, abnormalities in the retrochiasmatic region of the visual system, thalamic abnormalities, and cerebellar and cortical atrophy. Gliosis was divided into two groups: mild gliosis when no more than five scattered distinct, small $(2 \mathrm{~mm})$ areas of hyperintensity were present and extensive gliosis when larger or more than five areas of hyperintensity were present.

MRI findings were also classified into three groups: normal when no abnormalities were present or when a solitary finding such as an arachnoid cyst was found (group 1); mildly abnormal when mild gliosis, mild VD, irregularly shaped ventricles, corpus callosum thinning, or a combination of these was present (group 2); and severely abnormal when extensive gliosis or gliosis combined with marked VD was present (group 3). Thalamic lesions, an abnormal retrochiasmatic region of the visual system, and cerebellar and cortical atrophy were also classified as major abnormalities.

IQ

All children performed the following five subtests of the WISC-R (Dutch version): similarities, vocabulary, block design, picture arrangement, and digit span. They were supervised by a psychologist (AFL), unaware of their neonatal status. Using the procedures and tables published by Kaufman, ${ }^{11}$ scaled scores were converted to an estimated IQ score which is within a 95\% confidence interval of the full scale IQ score, with a standard error of estimate of 6.3.

\section{Motor function}

Motor performance was assessed by a physiotherapist (ICVH), blinded to MRI outcome. The Movement ABC contains three domains: manual dexterity (three items), ball skills (two items), and balance (three items). Each item is scored from 0 (best score) to 5 (poorest score). The total impairment score (TIS) is the sum of the subscores. Raw scores are converted to percentile scores and classified as follows: <p5 (definitely abnormal), between p5 and p15 (borderline), and >pl5 (normal).

The presence of CP was classified according to the Hagberg criteria. ${ }^{15}$

\section{Data analysis}

Descriptives of specific findings on cranial US and MRI were calculated. For each US group, predictive values and corresponding 95\% confidence intervals were calculated for inclusion in each of the three MRI groups. Mean IQ and median Movement ABC TIS were tested between US and MRI groups 1 and 2 and between groups 1 and 3 using $t$ tests and Mann-Whitney $U$ tests, respectively. Similar group comparisons were made for proportions with low versus normal IQ and for proportions in percentiles of Movement ABC using $\chi^{2}$ tests. SPSS (version 10.1; SPSS, Chicago, IL, USA) was used for all analyses.

\section{RESULTS}

\section{US findings}

Neonatal cranial US findings are listed in table 1. Ninety six infants were assigned to group 1 (normal US), 89 to group 2 (mildly abnormal US), and 36 to group 3 (severely abnormal US).

\section{MRI findings}

MRI findings at a median age of 8.1 years are listed in table 2 . Eighty seven children were assigned to group 1 (normal MRI), 104 to group 2 (mildly abnormal MRI), and 30 to group 3 (severely abnormal MRI).

\section{Association between US and MRI}

Table 3 shows the predictive values of US group classifications for MRI group classifications. Overall, there was poor correspondence between US and MRI. Those in US groups 1 and 2 had an approximately 50\% chance of being in the corresponding MRI groups. Despite poor overall correspondence, over $70 \%$ of those with major lesions on US had major lesions on MRI. There was only a $1 \%$ chance among those with a normal US of having a major lesion on MRI.

\section{IQ}

Mean IQ overall was 100 (table 4). Mean IQ in US group 1 was marginally higher than in group 2 but substantially and significantly higher than in group 3. Mean IQ in MRI group 1 
Table 1 Neonatal cranial US findings in a cohort of 221 preterm infants

\begin{tabular}{|c|c|}
\hline Normal, n (\%) & $92(41.6 \%)$ \\
\hline \multirow{6}{*}{$\mathrm{IVH}, \mathrm{n}(\%)$} & 79 (35.7\%) \\
\hline & IVH grade I: 11 (13.9\%) \\
\hline & IVH grade II: 48 (60.8\%) \\
\hline & IVH grade III: 9 (11.4\%) \\
\hline & IVH grade IV: 11 (13.9\%) \\
\hline & PHVD: 18 \\
\hline \multirow[t]{4}{*}{ PVL, n (\%) } & $41(18.6 \%)$ \\
\hline & PVL grade I: 32 (78.0\%) \\
\hline & PVL grade II: 4 (9.8\%) \\
\hline & PVL grade III: 5 (12.2\%) \\
\hline \multirow[t]{10}{*}{ Other, n (\%) } & $26(11.8 \%)$ \\
\hline & Convexity haemorrhage: 2 \\
\hline & Infarction: 2 \\
\hline & Thalamus lesion: 2 \\
\hline & Calcifications: 3 \\
\hline & Pseudocysts: 3 \\
\hline & Ventricular dilatation: 9 \\
\hline & GL cyst: 1 \\
\hline & GL necrosis: 1 \\
\hline & Plexus cyst: 3 \\
\hline
\end{tabular}

$\mathrm{GL}$, germinal layer; $I V H$, intraventricular haemorrhage; PHVD, post haemorrhagic ventricular dilatation; PVL, periventricular leukomalacia. It should be noted that 92 infants had a completely normal US; the other numbers do not add up to 129 due to multiple findings in some of the children.

was marginally but significantly higher than in group 2, but substantially and significantly higher compared to group 3 .

The proportion of children with an IQ $\leqslant 85$ was not different between US groups 1 and 2 but was significantly higher in US group 3. For MRI groups the same pattern was seen.

\section{Motor function}

Movement ABC tests resulted in a median overall TIS of 5.5 (table 5). Median TIS in US group 1 was not significantly different from group 2, but it was substantially and significantly different between US groups 1 and 3. In the MRI groups, median TIS between groups 1 and 2 and between groups 1 and 3 were both significantly different.

The proportions of children with definite motor problems $($ TIS $<\mathrm{p} 5$ ), borderline problems (TIS p5-15), and normal motor performance (TIS $>$ p15) were not significantly different between US and MRI groups 1 and 2. However, a significant difference between groups 1 and 3 for both US and MRI was found.

CP was present in 20 children: hemi/di/quadriplegia in six each and cerebellar ataxia in two. Only one child, with cerebellar ataxia, had a normal neonatal cranial US but a severely abnormal MRI. Of the remaining 19 children with CP, four had a mildly abnormal US (two VD, one IVH II, one IVH II with PVL I) and 15 had a severely abnormal US. Not one of the CP children had a normal MRI: three had a mildly abnormal MRI and 17 a severely abnormal MRI.

\section{DISCUSSION}

Neonatal cranial US and school age MRI were compared in 221 preterm infants. Almost half of the children with a normal US had a normal MRI, while the abnormalities seen
Table 2 MRI findings at school age in a cohort of 221 preterm infants

\begin{tabular}{|c|c|}
\hline Normal, n (\%) & $83(37.6 \%)$ \\
\hline Gliosis, n (\%) & $\begin{array}{l}64(29.0 \%) \\
\text { Mild gliosis: } 38(59.4 \%) \\
\text { Extensive gliosis: } 26(40.6 \%)\end{array}$ \\
\hline Ventricular dilatation, $\mathrm{n}(\%)$ & $\begin{array}{l}81 \text { (37.7\%) } \\
\text { Global: } 65(80.2 \%) \\
\text { Focal: } 16(19.8 \%)\end{array}$ \\
\hline $\begin{array}{l}\text { Abnormally shaped corpus } \\
\text { callosum, n (\%) }\end{array}$ & $\begin{array}{l}56(25.3 \%) \\
\text { Generalised thinning: } 19(33.9 \%) \\
\text { Focal thinning: } 37(66.1 \%)\end{array}$ \\
\hline $\begin{array}{l}\text { Abnormal retrochiasmatic } \\
\text { region of the visual } \\
\text { system, } n(\%)\end{array}$ & $8(3.6 \%)$ \\
\hline Thalamus abnormalities, n (\%) & $6(2.7 \%)$ \\
\hline Cerebellar atrophy, $\mathrm{n}(\%)$ & $3(1.4 \%)$ \\
\hline Cortical atrophy, $\mathrm{n}(\%)$ & $1(0.5 \%)$ \\
\hline Other, $n(\%)$ & $\begin{array}{l}9 \text { (4.1\%) } \\
\text { Pineal cyst: } 2 \\
\text { Arachnoidal cyst: } 3 \\
\text { Small pituitary gland: } 1 \\
\text { Extra cerebral CSF cyst: } 1 \\
\text { Mesencephalon lesion: } 1\end{array}$ \\
\hline
\end{tabular}

CSF, cerebral spinal fluid. It should be noted that 83 children had a completely normal MRI; the other numbers do not add up to 138 due to multiple findings in some of the children.

in the other half were usually very mild. In only one case was a major lesion (cerebellar atrophy and VD) diagnosed on MRI. Of the children with minor abnormalities on US, again almost half had a normal MRI and only 3.4\% had major lesions. However, over $70 \%$ of the children with major abnormalities on US had severe abnormalities on MRI. Two children with a severely abnormal cranial US (IVH III) had a completely normal school age MRI. Our data show that if cranial US is performed according to strict criteria, a normal US excludes severe lesions on later MRI in $99 \%$ of cases.

When considering these results, some matters should be taken into account. We performed a prospective, population based study in which all neonates with GA $<32$ weeks and/or $\mathrm{BW} \leqslant 1500 \mathrm{~g}$ had serial cranial US examinations according to a strict protocol. The inclusion percentage was high (78\%). We assessed the US according to the most severe lesions seen at any time in contrast to previous investigators who usually examined only one single US at a fixed moment. ${ }^{16}$

We have recently shown that performing sequential US scans is important as localised cysts (PVL II) may take a long time to develop and may have resolved by term age. ${ }^{17}$ If only one US is done at term age, cystic lesions may be missed. However, slight VD, irregular ventricular walls, and long $T_{2}$ suggestive of gliosis might be noticed on later MRI.

In our 221 children, we found no abnormalities on MRI in $37.6 \%$ of the cases. Skranes et al ${ }^{18}$ investigated 20 very low birthweight infants when aged 6 and a normal MRI was seen in only two children. Gliotic changes and VD were both seen in $60 \%$ in contrast to $29 \%$ with gliosis and $37.7 \%$ with VD in our group. These differences might be explained by the large variations in the sizes of the study groups and the fact that Skranes et al excluded children who had a normal MRI at the age of 1 year.

Table 3 Predictive values $(95 \% \mathrm{Cl})$ of ultrasound results for findings on MRI

\begin{tabular}{lccc}
\hline US/MRI & MRI group $\mathbf{1}, \mathbf{n}=\mathbf{8 7}$ & MRI group 2, $\mathbf{n = 1 0 4}$ & MRI group 3, $\mathbf{n = 3 0}$ \\
\hline US group 1, $\mathrm{n}=96$ & $45(46.9 ; 36.6$ to 57.3$)$ & $50(52.1 ; 41.6$ to 62.4$)$ & $1(1.0 ; 0.0$ to 5.7$)$ \\
US group 2, $\mathrm{n}=89$ & $40(44.9 ; 34.4$ to 55.9$)$ & $46(51.7 ; 40.8$ to 62.4$)$ & $3(3,4 ; 0.7$ to 9.5$)$ \\
US group 3, $\mathrm{n}=36$ & $2(5.6 ; 0.7$ to 18.7$)$ & $8(22.2 ; 10.1$ to 39.2$)$ & $26(72.2 ; 54.8$ to 85.8$)$ \\
\hline
\end{tabular}


Table $4 I Q$ at school age related to US and MRI groups

\begin{tabular}{|c|c|c|c|c|c|c|}
\hline US & Group $1, n=96$ & Group 2, $n=89$ & $p$ for difference $1-2$ & Group 3, n= 36 & $\mathrm{p}$ for difference $1-3$ & All, $n=221$ \\
\hline 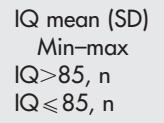 & $\begin{array}{l}102(13) \\
74-135 \\
85 \\
11\end{array}$ & $\begin{array}{l}101(13) \\
65-129 \\
77 \\
12\end{array}$ & $\begin{array}{l}0.39 \\
0.68\end{array}$ & $\begin{array}{l}94(18) \\
56-138 \\
23 \\
13\end{array}$ & $\begin{array}{l}0.005 \\
0.001\end{array}$ & $\begin{array}{l}100(14) \\
56-138 \\
185 \\
36\end{array}$ \\
\hline MRI & Group 1, $n=87$ & Group 2, n= 104 & $p$ for difference $1-2$ & Group 3, $n=30$ & $\mathrm{p}$ for difference $1-3$ & All, $n=221$ \\
\hline $\begin{array}{l}\mid Q \text { mean }(S D) \\
M i n-\max \\
I Q>85, n \\
I Q \leqslant 85, n\end{array}$ & $\begin{array}{l}104(13) \\
77-138 \\
80 \\
7\end{array}$ & $\begin{array}{l}100(13) \\
65-135 \\
88 \\
16\end{array}$ & $\begin{array}{l}0.026 \\
0.12\end{array}$ & $\begin{array}{l}91(16) \\
56-126 \\
17 \\
13\end{array}$ & $\begin{array}{l}<0.0001 \\
<0.0001\end{array}$ & $\begin{array}{l}100(14) \\
56-138 \\
185 \\
36\end{array}$ \\
\hline
\end{tabular}

Our data are more in agreement with two other studies. ${ }^{19} 20$ In the first study, $42.5 \%$ of 87 children showed abnormalities on MRI at $15-17$ years of age. In the second study $(n=72)$, 40 children had definitely abnormal and 15 had equivocal MRI scans at 14-15 years of age. Similar to the first study, abnormalities in the white matter, the size of the lateral ventricles, and the corpus callosum were especially common. Of the 59 children with a normal or mildly abnormal neonatal US scan, 13 had equivocal and 30 definite abnormalities on MRI. Our better correlation between major US abnormalities and severe lesions on later MRI can probably be explained by the use of a mechanical sector scanner with a 7.5 $\mathrm{MHz}$ transducer instead of a linear array machine with a $5-7 \mathrm{MHz}$ transducer, which is less well suited for the detection of lesions in the periventricular white matter.

Although a normal US almost excluded severe lesions on later MRI and a severely abnormal US correlated well with severe lesions on later MRI, there was little correspondence between normal and mildly abnormal US and MRI groups. This is most likely due to poor detection of subtle white matter lesions with cranial US. ${ }^{7-9}$ In a recent editorial, Volpe ${ }^{21}$ highlighted the importance of diffuse white matter injury with subsequently impaired white matter development. Infants with a normal cranial US could subsequently develop subtle gliotic changes and infants with PVL I could either normalise or show persistent changes in the white matter, seen as gliotic changes or mild VD on later MRI. Counsell et $a^{22}$ carried out a diffusion weighted MRI study in preterm infants at term equivalent and showed that diffuse excessive high signal intensity (DEHSI) represents diffuse white matter anomalies. It is possible that the US equivalent of DEHSI is prolonged flaring, like in PVL I. A large proportion of our mildly abnormal US group consisted of infants with an IVH I or II. In the absence of associated PVL I, a normal MRI is to be expected. Kuban $e t$ al $^{23}$ suggested from their US data in
1605 infants that IVH and VD were powerful predictors of white matter damage, both localised as well as diffuse. However, no MRI study was performed to confirm this.

Recently, more sophisticated techniques such as volume measurements, ${ }^{24-26}$ diffusion tensor imaging, ${ }^{27}$ and $\mathrm{fMRI}^{28}$ have become available. Our study is limited in the sense that only conventional MRI techniques were used. However, our aim was to evaluate the effect of neonatal US lesions on subsequent brain development.

One of the main clinical reasons for examining the neonatal brain is to predict neuromotor development. Two parameters for outcome (IQ and motor performance) were measured in this study. Whether a child was in the normal or the mildly abnormal US group did not have an effect on mean IQ. Mean IQ was only significantly decreased with major abnormalities on US, but the range of IQ in US group 3 was considerable. MRI at school age appeared to be more accurate in the prediction of learning abilities. However, the clinical relevance of a statistically significant difference of 4 IQ points between MRI groups 1 and 2 can be questioned. There were no significant differences in the number of children with a low IQ $(\leqslant 85)$ between either US or MRI groups 1 and 2. Both the US and MRI group 3 had a significantly higher number of children with an IQ $\leqslant 85$.

The same pattern was seen for motor function: US group 1 did not differ in median TIS from group 2 but was significantly better than US group 3. MRI group 1 scored significantly better in median TIS compared with MRI groups 2 and 3. Although very significant as regards $p$ value, $a$ median TIS of 4 in MRI group 1 is similar in daily practice to a TIS of 6.25 in MRI group 2. The number of children with serious motor problems was not significantly different between US and MRI groups 1 and 2 .

We conclude that a normal neonatal cranial US, if obtained in a systematic way within regular time intervals, excludes a severely abnormal MRI at school age in $99 \%$ of cases.

Table 5 Movement $A B C$ at school age related to US and MRI groups

\begin{tabular}{|c|c|c|c|c|c|c|}
\hline US & Group 1, n=96 & Group 2, $n=89$ & $p$ for difference $1-2$ & Group 3, $n=36$ & $\mathrm{p}$ for difference $1-3$ & All $(n=221)$ \\
\hline TIS median & 5.25 & 5.0 & & 15.75 & & 5.5 \\
\hline Min-max & $0-36.5$ & $0-40$ & 0.67 & $0.5-40$ & $<0.0001$ & $0-40$ \\
\hline Mov $A B C<p 5, n$ & 6 & 12 & & 19 & & 37 \\
\hline Mov ABC p5-p15, n & 10 & 6 & 0.19 & 5 & $<0.0001$ & 21 \\
\hline Mov $A B C>p 15, n$ & 80 & 71 & & 12 & & 163 \\
\hline MRI & Group 1, n= 87 & Group 2, n= 104 & p for difference $1-2$ & Group 3, n= 30 & $\mathrm{p}$ for difference $1-3$ & All $(n=221)$ \\
\hline TIS median & 4.0 & 6.25 & & 19.25 & & 5.5 \\
\hline Min-max & $0-21$ & $0-40$ & $<0.0001$ & $2.5-40$ & $<0.0001$ & $0-40$ \\
\hline Mov $A B C<p 5, n$ & 4 & 14 & & 19 & & 37 \\
\hline Mov ABC p5-15, n & 7 & 10 & 0.095 & 4 & $<0.0001$ & 21 \\
\hline Mov $A B C>p 15, n$ & 76 & 80 & & 7 & & 163 \\
\hline
\end{tabular}

Mov, Movement; TIS, total impairment score. 


\section{What is already known on this topic}

- Neonatal cranial ultrasound is reliable for the diagnosis of germinal matrix/intraventricular haemorrhage and cystic periventricular leukomalacia

- Cranial ultrasound is not the best imaging technique to detect subtle white matter lesions

\section{What this study adds}

- In a large cohort, a good correlation between severe lesions on neonatal cranial ultrasound and childhood MRI was found

- Mild MRI abnormalities rather than mild ultrasound abnormalities were associated with poorer neurodevelopmental outcome

However, half of the children with normal US do have minor abnormalities on MRI. MRI findings correlated better than US with mean IQ and median TIS at school age. Although significant, these differences appear not to be clinically relevant for normal and mildly abnormal MRI groups. Subtle white matter lesions are better detected with MRI, which could explain the stronger correlation of MRI with neurodevelopmental outcome.

More sophisticated MRI techniques or MRI in selected patients may show even better differentiation but further research will be needed to support this assumption.

\section{ACKNOWLEDGMENTS}

The authors would like to thank the MRI technicians for examining the children.

\section{Authors' affiliations}

K J Rademaker, C S P M Uiterwaal, F J A Beek, I C van Haastert, A F Lieftink, F Groenendaal, D E Grobbee, L S de Vries, University Medical Centre Utrecht, Utrecht, The Netherlands

The University Medical Center Utrecht provided financial support (Zonproject) for this study

Competing interests: none declared

\section{REFERENCES}

1 de Vries LS, Eken $\mathrm{P}$, Beek E, et al. The posterior fontanelle: a neglected acoustic window. Neuropediatrics 1996;27:101-4.

2 de Vries LS, Van Haastert IL, Rademaker KJ, et al. Ultrasound abnormalities preceding cerebral palsy in high-risk preterm infants. J Pediatr 2004; 144:815-20.

3 Roelants-van Rijn AM, Groenendaal F, Beek FJ, et al. Parenchymal brain injury in the preterm infant: comparison of cranial ultrasound, MRI and neurodevelopmental outcome. Neuropediatrics 2001;32:80-9.
4 Robertson NJ, Wyatt JS. The magnetic resonance revolution in brain imaging: impact on neonatal intensive care. Arch Dis Child Fetal Neonatal Ed 2004;89:F193-7.

5 de Vries LS, Rademaker KJ, Groenendaal F, et al. Correlation between neonatal cranial ultrasound, MRI in infancy and neurodevelopmental outcome in infants with a large intraventricular haemorrhage with or without unilateral parenchymal involvement. Neuropediatrics 1998;29:180-8.

6 Maalouf EF, Duggan PJ, Counsell SJ, et al. Comparison of findings on cranial ultrasound and magnetic resonance imaging in preterm infants. Pediatrics 2001;107:719-27.

7 Miller SP, Cozzio CC, Goldstein RB, et al. Comparing the diagnosis of white matter injury in premature newborns with serial MR imaging and transfontanel ultrasonography findings. AJNR Am J Neuroradiol 2003;24:1661-9.

8 Inder TE, Anderson NJ, Spencer C, et al. White matter injury in the premature infant: a comparison between serial cranial sonographic and MR findings at term. AJNR Am J Neuroradiol 2003;24:805-9.

9 Debillon T, N'Guyen S, Muet A, et al. Limitations of ultrasonography for diagnosing white matter damage in preterm infants. Arch Dis Child Fetal Neonatal Ed 2003;88:F275-9.

10 Childs AM, Cornette L, Ramenghi LA, et al. Magnetic resonance and cranial ultrasound characteristics of periventricular white matter abnormalities in newborn infants. Clin Radiol 2001;56:647-55

11 Kaufman AS. Intelligent testing with the WISC-R. New York: Wiley, 1979.

12 Croce RV, Horvat M, McCarthy E. Reliability and concurrent validity of the movement assessment battery for children. Percept Mot Skills 2001;93:275-80.

13 Papile LA, Burstein J, Burstein R, et al. Incidence and evolution of subependymal and intraventricular hemorrhage: a study of infants with birth weights less than 1,500 gm. J Pediatr 1978;92:529-34.

14 de Vries LS, Eken P, Dubowitz LM. The spectrum of leukomalacia using cranial ultrasound. Behav Brain Res 1992;49:1-6.

15 Hagberg B, Hagberg G, Olow I. The changing panorama of cerebral palsy in Sweden 1954-1970. I. Analysis of the general changes. Acta Paediatr Scand 1975;64:187-92.

16 Valkama AM, Paakko EL, Vainionpaa LK, et al. Magnetic resonance imaging at term and neuromotor outcome in preterm infants. Acta Paediatr 2000;89:348-55

17 Pierrat V, Duquennoy C, van Haastert IC, et al. Ultrasound diagnosis and neurodevelopmental outcome of localised and extensive cystic periventricular leucomalacia. Arch Dis Child Fetal Neonatal Ed 2001;84:F151-6.

18 Skranes JS, Nilsen G, Smevik O, et al. Cerebral MRI of very low birth weight children at 6 years of age compared with the findings at 1 year. Pediatr Radiol 1998;28:471-5.

19 Cooke RW, Abernethy $\sqcup$. Cranial magnetic resonance imaging and school performance in very low birth weight infants in adolescence. Arch Dis Child Fetal Neonatal Ed 1999;81:F1 16-21.

20 Stewart AL, Rifkin L, Amess PN, et al. Brain structure and neurocognitive and behavioural function in adolescents who were born very preterm. Lancet 1999;353:1653-7.

21 Volpe JJ. Cerebral white matter injury of the premature infant-more common than you think. Pediatrics 2003;112:176-80.

22 Counsell SJ, Allsop JM, Harrison MC, et al. Diffusion-weighted imaging of the brain in preterm infants with focal and diffuse white matter abnormality. Pediatrics 2003;112:1-7.

23 Kuban K, Sanocka U, Leviton A, et al. White matter disorders of prematurity: association with intraventricular hemorrhage and ventriculomegaly. The Developmental Epidemiology Network. J Pediatr 1999;134:539-46.

24 Abernethy L, Cooke RW, Foulder-Hughes L. Caudate and hippocampal volumes, intelligence, and motor impairment in 7-year-old children who were born preterm. Pediatr Res 2004;55:884-93.

25 Abernethy L, Palaniappan M, Cooke RW. Quantitative magnetic resonance imaging of the brain in survivors of very low birth weight. Arch Dis Child 2002;87:279-83.

26 Nosarti C, Al Asady MH, Frangou S, et al. Adolescents who were born very preterm have decreased brain volumes. Brain 2002;125:1616-23.

27 Nagy Z, Westerberg H, Skare S, et al. Preterm children have disturbances of white matter at 11 years of age as shown by diffusion tensor imaging. Pediatr Res 2003;54:672-9.

28 Santhouse AM, Ffytche DH, Howard RJ, et al. The functional significance of perinatal corpus callosum damage: an fMRI study in young adults. Brain 2002;125:1782-92. 